\title{
DNA hypomethylation of interleukin 8 in clear cell renal cell carcinoma
}

\author{
KOO HAN YOO ${ }^{1}$, YONG-KOO PARK ${ }^{2}$ and SUNG-GOO CHANG ${ }^{1}$ \\ Departments of ${ }^{1}$ Urology and ${ }^{2}$ Pathology, School of Medicine, Kyung Hee University, \\ Seoul 130-702, Republic of Korea
}

Received July 16, 2012; Accepted October 10, 2012

DOI: $10.3892 / \mathrm{ol} .2012 .992$

\begin{abstract}
DNA hypomethylation is associated with carcinogenesis due to its involvement in cancer initiation and progression. In this study we analyzed the hypomethylation status of candidate genes in clear cell renal cell carcinoma (CCRCC) using a large-scale, high-throughput DNA methylation profiling technique. Results revealed that hypomethylated interleukin 8 (IL8) gene was the most prominent of the candidate genes, with a beta value difference of 0.406 (cancer tissue mean beta value, 0.346; normal tissue mean beta value, 0.752). Validation results using sequencing analysis demonstrated that the methylation rate was $2.4 \%$ in cancer tissue and $14.7 \%$ in normal tissue, whilst the non-methylation rate was $82.9 \%$ in cancer tissue and $52.9 \%$ in normal tissue. However, the hypomethylation status of IL8 had no significant relationship with Fuhrman's nuclear grade, tumor node metastasis (TNM) stage or survival ( $\mathrm{P}>0.05)$. We revealed that the IL8 gene is maximally hypomethylated in cancer tissue compared to normal tissue.
\end{abstract}

\section{Introduction}

Interleukin 8 (IL8) is produced by macrophages, neutrophils, endothelial cells and cancer cells. It is a proinflammatory chemokine associated with the promotion of neutrophil chemotaxis and degranulation (1). This chemokine activates intracellular signaling pathways using $G$ protein-coupled receptors (1). A number of chemokines, such as IL8, promote and regulate neoplastic progression, including angiogenesis and metastasis (2). IL8 is a potent angiogenic factor and controls the expression of vascular endothelial growth factor (VEGF) in endothelial cells (3). Overexpression of VEGF is closely associated with the progression of clear cell renal cell carcinoma (CCRCC) (4).

Correspondence to: Professor Sung-Goo Chang, Department of Urology, Kyung Hee University Hospital, 1 Hoegi-dong, Dongdaemun-ku, Seoul 130-702, Republic of Korea

E-mail: sgchang@khu.ac.kr

Key words: carcinoma, renal cell, DNA methylation, interleukin 8
Hypomethylation of genomic DNA has been associated with increased rates of genomic instability (5). Hypomethylation of $\mathrm{CpG}$ dinucleotides in genomic DNA is known as one of the somatic epigenetic alterations identified in human cancers (5). DNA hypomethylation is postulated to affect carcinogenesis due to its involvement in cancer initiation and progression (6). In this study, we analyzed the hypomethylation status of candidate genes, including IL8, in CCRCC using a large-scale, high-throughput DNA methylation profiling technique. The correlation between the hypomethylation status of the candidate gene (IL8) and clinicopathological parameters was examined.

\section{Material and methods}

Tissue specimens consisted of 46 cancer tissues and 46 matched adjacent normal tissues from CCRCC patients who had undergone nephrectomy at Kyung Hee University Hospital between 1999 and 2005. The study was approved by the institutional review board at Kyung Hee University Medical Center. Preparation of DNA samples and DNA extraction were implemented as previously described (7). Bisulfite conversion of all DNA samples was performed with EZ-96 DNA Methylation kit (Zymo Research, Orange, CA, USA). The methylcytosine content was quantified using a GoldenGate Methylation Cancer Panel I microarray (Illumina, San Diego, CA, USA) (8). Following the bisulfite conversion and methylation chip assay, technical replicates were prepared for each sample using the same converted DNA. Beta values for matching normal and cancer clinical samples were compared; differential methylation was readily detected (8). The methylation status of $\mathrm{CpG}$ sites was examined by bisulfite sequencing according to the protocol employed by Herman et al (9). The methylation status of the $1505 \mathrm{CpG}$ sites was measured using a data matrix of beta values. An additional filter based on a Student's t-test was applied. This required a maximal difference in beta value between the two groups (10). The hypomethylation status and clinicopathological characteristics of CCRCC patients were analyzed.

\section{Results}

We identified one marker (IL8) out of 807 cancer-related candidate genes (Fig. 1). Results revealed that the mean beta value difference of IL8 was 0.406 (cancer tissue mean beta 
Table I. Baseline characteristic of clear cell renal cell carcinoma (CCRCC) patients.

\begin{tabular}{lccr}
\hline & Weak hypomethylation $(\mathrm{n}=23)$ & Strong hypomethylation $(\mathrm{n}=23)$ & P-value \\
\hline Age & $61.30 \pm 11.166$ & $62.74 \pm 10.618$ & 0.502 \\
Gender & & & 0.738 \\
Male & 18 & 7 & \\
Female & 5 & & \\
Fuhrman's nuclear grade & & 2 & \\
I & 2 & 7 & 0.316 \\
II & 11 & 9 & \\
III & 7 & 5 & 0.695 \\
IV & 3 & 15 & \\
TNM stage & & 4 & 1.000 \\
T1 & 16 & 4 & \\
T2 & 4 & 0 & 0.386 \\
T3 & 3 & 22 & \\
T4 & 0 & 1 & \\
N0 & 22 & 4 & \\
N1 & 1 & 19 & \\
M0 & 21 & & \\
M1 & 2 & & \\
\hline
\end{tabular}

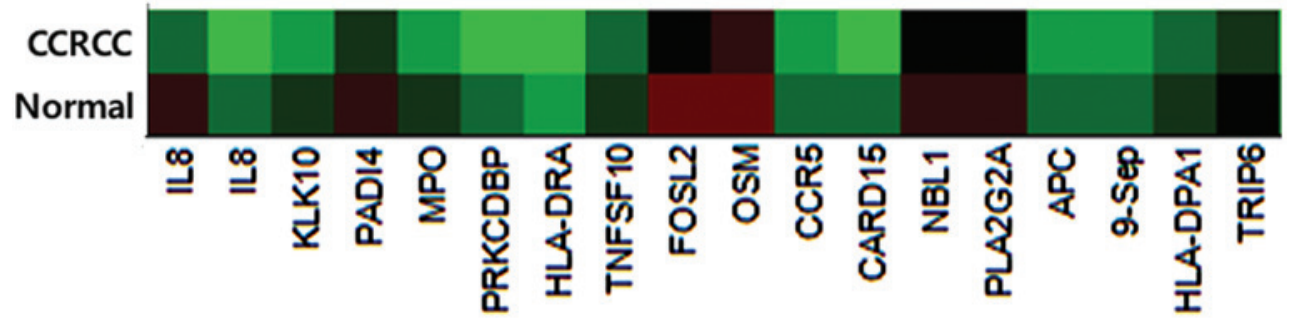

Figure 1. Heat map analysis of clear cell renal cell carcinoma (CCRCC) and normal samples. Green and red colors represent low and high methylation levels, respectively. Of 807 cancer related genes, interleukin 8 (IL8) had the largest beta value difference (-0.406) between cancer and normal tissue.

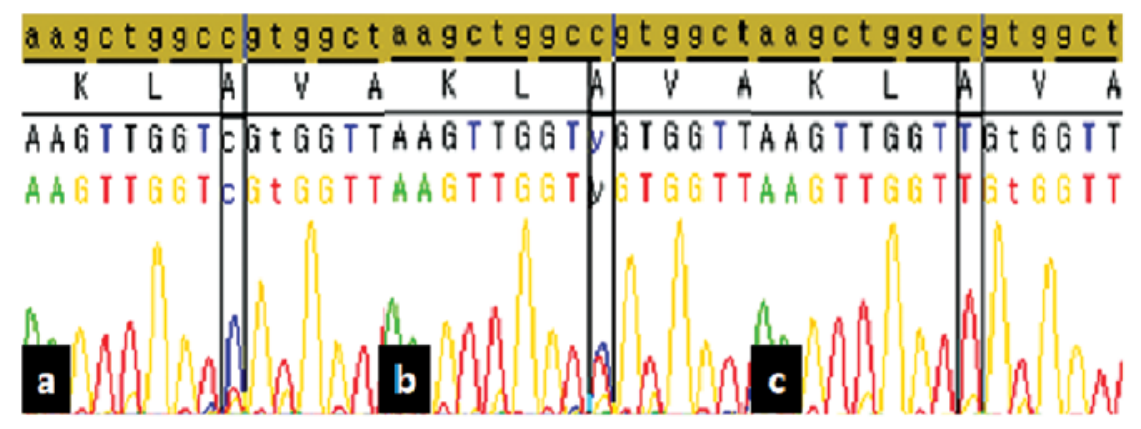

Figure 2. Sequencing analysis of abnormal methylation in the promoter region of interleukin 8 (IL8). (a) Methylated CpG islands in the cancer portion; the Cs of the $\mathrm{CpG}$ islands remain unchanged after modification by sodium bisulfite. (b) Heterogenous CpG islands; mixed methylated and unmethylated CpG islands . (c) Unmethylated CpG islands in which Cs become Ts following modification with sodium bisulfite.

value, 0.346; normal tissue mean beta value, 0.752). Of the 46 CCRCC patients, two groups were defined according to their beta value difference; weak hypomethylation (a difference smaller than the mean value) and strong hypomethylation (a difference greater than the mean value). Table I indicates that the hypomethylation status of IL8 had no significant correlation with either Fuhrman's nuclear grade or tumor node metastasis $(\mathrm{TNM})$ stage $(\mathrm{P}>0.05)$. 


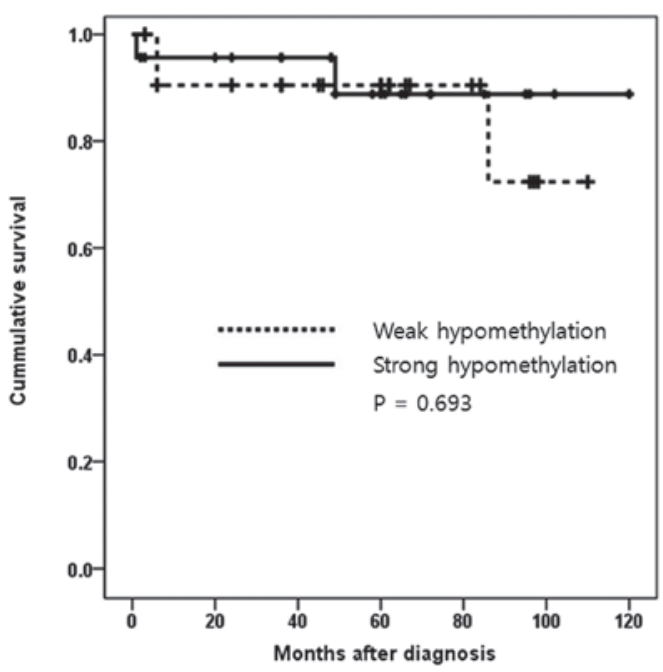

Figure 3. Kaplan-Meier curves of overall survival in clear cell renal cell carcinoma (CCRCC) patients by interleukin 8 (IL8) methylation status.

We examined the methylation status of the $\mathrm{CpG}$ site by bisulfite sequencing. Fig. 2 reveals the hypomethylation results of IL8 using bisulfite sequencing. Validation results using sequencing analysis demonstrated that the methylation rate was $2.4 \%$ in cancer tissue and $14.7 \%$ in normal tissue, whilst the non-nmethylation rate was $82.9 \%$ in cancer tissue and $52.9 \%$ in normal tissue. The overall survival time of CCRCC patients was analyzed according to either a weak or strong hypomethylation status of IL8 (Fig. 3). There was no statistical influence of IL8 hypomethylation status on survival, as demonstrated by the Kaplan-Meier survival curve $(\mathrm{P}=0.693)$.

\section{Discussion}

In this study, beta value differences of cancer and normal tissue were not associated with clinicopathological status. However, DNA hypomethylation of IL8 was widely detected in cancer compared to normal kidney tissue. Alteration of genomic DNA methylation, such as hyper- and hypomethylation, affects human tumorigenesis (5). Hypermethylation of promoter $\mathrm{CpG}$ islands is capable of inactivating important tumor-suppressor genes (11). Hypomethylation of genomic DNA has been associated with increased rates of genomic instability $(6,12)$.

Suzuki et al demonstrated that genomic DNA hyper- and hypomethylation alterations in gastrointestinal cancer were distributed gradually and increased with cancer patient age (13). It was concluded that age-dependent accumulation of DNA demethylation precedes diploidy loss in gastrointestinal cancer. Cadieux et al demonstrated that hypomethylation of Sat2 sequences is associated with copy number alterations of the adjacent euchromatin in human glioblastomas (14). This implicated hypomethylation to be a predisposing factor for specific genetic alterations in glioblastoma. The analysis between DNA hypomethylation and prostate cancer was reported by Yegnasubramanian et al, who performed a tiered gene expression microarray and bisulfite genomic sequencing-based approach to identify the methyla- tion status of prostate cancer (5). The authors demonstrated that a class of cancer testis antigen genes undergoes $\mathrm{CpG}$ island hypomethylation and overexpression in primary and metastatic prostate cancer. DNA hypomethylation patterns were heterogenous across different metastatic sites within the same patients.

IL8 is a proinflammatory cytokine for leukocytes and is involved in tumor growth, metastasis and survival of solid organ cancer $(15,16)$. Certain studies have analyzed IL8 methylation status in human cancer $(17,18)$. Dimberg et al revealed the protein expression of IL8 in plasma, tumor and paired normal tissue, and the methylation status of the IL8 gene in colorectal cancer (17). The authors demonstrated that a significantly higher level of IL8 was present in cancer tissue compared to normal tissue. IL8 hypomethylation was detected in $64 \%$ of the cancer tissue, whereas no hypomethylation was found in the paired normal tissue. De Larco et al analyzed the correlation between the metastatic potential of breast carcinoma cell lines and the ectopic expression of IL8 (18). The authors revealed that an aberrant methylation pattern may be responsible for the differences in IL8 between high and low metastatic cell lines. Two CpG sites of IL8 were fully methylated in the high metastatic cell lines. However, these cell lines produced large quantities of IL8. The authors suggested that there may be additional epigenetic control mechanisms that have not yet been fully appreciated or explored.

In summary, this study demonstrated that the IL8 gene was maximally hypomethylated in CCRCC cancer tissue compared to normal tissue. However, levels of DNA hypomethylation were not correlated with the clinicopathological status of the patient.

\section{Acknowledgements}

This study was supported by a grant from the Korea Science and Engineering Foundation (KOSEF), funded by the Korean government (MOST) (No. R13-2002-020-02001-0, 2007).

\section{References}

1. Waugh DJ and Wilson C: The interleukin-8 pathway in cancer. Clin Cancer Res 14: 6735-6741, 2008.

2. Vandercappellen J, Van Damme J and Struyf S: The role of CXC chemokines and their receptors in cancer. Cancer Lett 267: 226-244, 2008

3. Martin D, Galisteo R and Gutkind JS: CXCL8/IL8 stimulates vascular endothelial growth factor (VEGF) expression and the autocrine activation of VEGFR 2 in endothelial cells by activating NFkappaB through the CBM (Carma3/Bcl10/Malt1) complex. J Biol Chem 284: 6038-6042, 2009.

4. Djordjevic G, Mozetic V, Mozetic DV, et al: Prognostic significance of vascular endothelial growth factor expression in clear cell renal cell carcinoma. Pathol Res Pract 203: 99-106, 2007.

5. Yegnasubramanian S, Haffner MC, Zhang Y, et al: DNA hypomethylation arises later in prostate cancer progression than $\mathrm{CpG}$ island hypermethylation and contributes to metastatic tumor heterogeneity. Cancer Res 68: 8954-8967, 2008.

6. Karpf AR and Matsui S: Genetic disruption of cytosine DNA methyltransferase enzymes induces chromosomal instability in human cancer cells. Cancer Res 65: 8635-8639, 2005.

7. Kim GY, Park JH, Kim YW, Jung WW, Unni KK and Park YK: Absence of amplification of HER-2/neu (c-erbB-2) gene in Ewing's sarcoma: a real-time polymerase chain reaction method. Pathol Res Pract 200: 663-667, 2004.

8. Bibikova M, Lin Z, Zhou L, et al: High-throughput DNA methylation profiling using universal bead arrays. Genome Res 16: 383-393, 2006. 
9. Herman JG, Graff JR, Myöhänen S, Nelkin BD and Baylin SB: Methylation-specific PCR: a novel PCR assay for methylation status of CpG islands. Proc Natl Acad Sci USA 93: 9821-9826, 1996.

10. Yoo KH, Park YK, Kim HS, Jung WW and Chang SG: Epigenetic inactivation of HOXA5 and MSH2 gene in clear cell renal cell carcinoma. Pathol Int 60: 661-666, 2010.

11. Jones PA and Laird PW: Cancer epigenetics comes of age. Nat Genet 21: 163-167, 1999.

12. Eden A, Gaudet F, Waghmare A and Jaenisch R: Chromosomal instability and tumors promoted by DNA hypomethylation. Science 300: 455, 2003.

13. Suzuki K, Suzuki I, Leodolter A, et al: Global DNA demethylation in gastrointestinal cancer is age dependent and precedes genomic damage. Cancer Cell 9: 199-207, 2006.

14. Cadieux B, Ching TT, VandenBerg SR and Costello JF Genome-wide hypomethylation in human glioblastomas associated with specific copy number alteration, methylenetetrahydrofolate reductase allele status, and increased proliferation. Cancer Res 66: 8469-8476, 2006.
15. Xie K: Interleukin- 8 and human cancer biology. Cytokine Growth Factor Rev 12: 375-391, 2001.

16. Strieter RM, Belperio JA, Phillips RJ and Keane MP: CXC chemokines in angiogenesis of cancer. Semin Cancer Biol 14: 195-200, 2004.

17. Dimberg J, Ström K, Löfgren S, Zar N, Lindh M and Matussek A: DNA promoter methylation status and protein expression of interleukin-8 in human colorectal adenocarcinomas. Int J Colorectal Dis 27: 709-714, 2011.

18. De Larco JE, Wuertz BR, Yee D, Rickert BL and Furcht LT: Atypical methylation of the interleukin- 8 gene correlates strongly with the metastatic potential of breast carcinoma cells. Proc Natl Acad Sci USA 100: 13988-13993, 2003. 